\title{
CORRIGENDUM: ALGORITHMIC ENUMERATION OF IDEAL CLASSES FOR QUATERNION ORDERS*
}

\author{
MARKUS KIRSCHMER ${ }^{\dagger}$ AND JOHN VOIGHT VH $^{\ddagger}$
}

Abstract. This note corrects a mathematical error in the article "Algorithmic enumeration of ideal classes for quaternion orders" [SIAM J. Comput., 39 (2010), pp. 1714-1747].

DOI. $10.1137 / 120866063$

There is an error in the article [2] by the authors: The proof of Theorem B uses a diameter bound (7.1) of Chung [1],

$$
D(G) \leq\left\lceil\frac{\log (H-1)}{\log (k / \lambda)}\right\rceil,
$$

which holds for a $k$-regular directed graph $G$ of size $H$ with adjacency matrix $T$ and second-largest eigenvalue $\lambda$ under the hypotheses that (1) $k$ is an eigenvalue of $T$ with multiplicity 1 , and (2) $T$ is normal.

We apply this general diameter bound to a graph whose adjacency matrix $T$ represents the action of a Hecke operator on the space of Hilbert modular forms of level $\mathfrak{N}$ over some number field $F$.

Then, hypothesis (1) holds only when $F$ has strict class number 1, and (2) holds only when the sizes of the norm-one unit groups of all Eichler orders of level $\mathfrak{N}$ in a suitable quaternion algebra are equal. But since the adjacency matrix $T$ is in fact normal with respect to a (scaled) inner product slightly different than the standard one, we can correct the bound in a way which works generally and give a precise runtime analysis. The statement of the theorems remains unchanged. A corrected version of the paper can be obtained from the arXiv [3] or from the authors' home pages.

The authors sincerely regret this mistake and would like to thank Daniel Smertnig whose questions led to its discovery.

\section{REFERENCES}

[1] F. R. K. Chung, Diameters and eigenvalues, J. Amer. Math. Soc., 2 (1989), pp. 187-196.

[2] M. Kirschmer and J. Voight, Algorithmic enumeration of ideal classes for quaternion orders, SIAM J. Comput., 39 (2010), pp. 1714-1747.

[3] M. Kinschmer and J. Voight, Algorithmic enumeration of ideal classes for quaternion orders, corrected version, http://arxiv.org/abs/0808.3833.

\footnotetext{
* Received by the editors February 15, 2012; accepted for publication (in revised form) April 13, 2012; published electronically June 28, 2012.

http://www.siam.org/journals/sicomp/41-3/86606.html

$\dagger$ Lehrstuhl D für Mathematik, RWTH Aachen University, 52062 Aachen, Germany (Markus. Kirschmer@math.rwth-aachen.de).

${ }^{\ddagger}$ Department of Mathematics and Statistics, University of Vermont, Burlington, VT 05401 (jvoight@gmail.com).
} 